\title{
Quelques souhaits à propos des thèses de didactique des sciences physiques
}

\section{(2) OpenEdition \\ Journals}

Édition électronique

URL : http://journals.openedition.org/trema/3009

DOI : 10.4000/trema.3009

ISSN : 2107-0997

Éditeur

Faculté d'Éducation de l'université de Montpellier

\section{Édition imprimée}

Date de publication : 1 mai 1993

Pagination : 157-160

ISSN : 1167-315X

Référence électronique

"Quelques souhaits à propos des thèses de didactique des sciences physiques », Tréma [En ligne], 3-4 | 1993, mis en ligne le 29 novembre 2013, consulté le 21 avril 2019. URL : http:// journals.openedition.org/trema/3009; DOI : 10.4000/trema.3009

Ce document a été généré automatiquement le 21 avril 2019

Trema 


\section{Quelques souhaits à propos des thèses de didactique des sciences physiques}

1 La période pendant laquelle un chercheur prépare une thèse est souvent l'une des plus, sinon la plus, productive(s) de sa vie professionnelle de recherche. Les résultats de ce travail doivent donc être particulièrement utilisables (ce mot n'a aucun caractère restrictif : il ne signifie pas « utilitaires », ni même forcément "rapidement utilisables »). On n'est donc ni dans le registre de l'exercice de style, ni dans celui du contrôle des connaissances, mais dans celui de l'apport d'information (là encore, aucun caractère restrictif dans ce terme : il ne s'agit évidemment pas de livrer des observations brutes). Il faut redire ici que la didactique est une science expérimentale.

Il nous semble souhaitable que la rédaction fasse apparaître clairement :

La (ou les) questions qu'on s'est posée(s) au départ, et éventuellement celles qui ont surgi ou ont dû être reformulées en cours de route. Cette, ou ces, formulations de questions (certains préfèrent le terme « d'interrogations » pour en souligner le caractère explicitement analysé) se font compte tenu d'un certain nombre de spécifications. Cellesci peuvent relever de plusieurs niveaux, par exemple :

3 - Celles qui situent la question soulevée, sans pour autant intervenir directement dans le travail.

4 Ainsi : « je cherche à connaître les idées des personnes de la catégorie $\mathrm{X}$ sur tel point $\mathrm{Y}$ parce que je pense, a priori, que ces idées sont déterminantes dans un apprentissage ultérieur lors d'un enseignement $Z$ que je spécifie (plus ou moins) ».

$\mathrm{Ou}:$ «je cherche à spécifier les caractères d'un enseignement cohérent orienté vers tel but pour tel public, qui soit compatible avec telles contraintes [...], parce que je pense que cela présente tel type d'intérêt... ».

On appelle souvent cela le contexte pédagogique et idéologique de la recherche.

7 - Hypothèse qui détermine largement la structure du travail, sans être en elle-même facilement invalidable. 
8 Ainsi : « je fais l'hypothèse qu'il existe une cohérence dans les idées dites souvent « nonscientifiques » des élèves. Je vais donc chercher les lignes de forces de cette cohérence à travers des ensembles de situations d'interrogation. Si je ne trouve pas, je n'ai rien prouvé, sinon que ces lignes de cohérence, si elles existent, sont parfois difficiles à mettre en évidence. »

$\mathrm{Ou}:$ «je fais l'hypothèse que la connaissance peut être utilement décrite selon deux axes : déclaratif et procédural. Je construis mon exploration en conséquence... ». - Hypothèse plus spécifique, et plus susceptible d'être invalidée.

11 Ainsi : "les élèves de catégorie $\mathrm{X}$ ont souvent un raisonnement mononotionel en électrocinétique».

12 (Il n'est pas nécessaire d'inclure a posteriori, donc artificiellement, les résultats obtenus dans les hypothèses de départ, qui se voient ainsi toutes confirmées).

Le terme de "problématique " ne suffit pas à situer le statut de ce que l'on trouve dans le chapitre correspondant. Il nous semble utile de tendre vers une certaine précision à cet égard, sans tomber pour autant dans une taxonomie rigide d'un nouveau genre.

En bref, les hypothèses de travail doivent être clairement énoncées. Les unes sont l'objet même du travail. D'autres proviennent d'options, de théories, et parfois d'éclairages d'autres disciplines qui permettent de mieux décrire un contexte et d'affiner l'analyse. Celles-ci ne doivent pas envahir la thèse, au risque de la transformer en un travail d'épistémologie, psychologie ou sociologie amateur. Les théories didactiques ne sont pas encore suffisamment affirmées pour qu'une thèse puisse s'enfermer dans l'une d'elles. Mais il est souhaitable que l'élaboration de la problématique montre que les enjeux théoriques sont connus. Les concepts de didactique doivent être utilisés chaque fois qu'ils se révèlent utiles et qu'ils sont l'objet d'un consensus, mais précisés lorsqu'ils sont encore en cours de constitution. Les hypothèses non invalidables doivent être présentées comme telles.

\section{L'état de la question avant le début de la recherche.}

16 Tout le monde s'accorde sur l'importance de cette composante. Mais il est parfois impératif de doser l'encyclopédisme. C'est encore le critère d'articulation avec le travail effectué qui doit prédominer, et le souci de situer celui-ci dans un contexte d'acquis de recherche.

17 A ce jour, qu'est-ce que notre communauté entend par «acquis »? Ceux-ci se trouvent dans les thèses de didactique (françaises et étrangères), les rapports de recherche, les journaux tels que International Journal of Science Education, Cognitive Science etc. En cela, la didactique s'aligne sur toutes les disciplines de recherche.

18 Les méthodes d'investigations et les raisons qui ont présidé à leur choix. Il n'y a pas lieu de refaire ici un cours de méthodologie. Mais il semble utile de souligner l'intérêt de mettre en œuvre, dans la mesure du possible, des méthodes d'investigation facilement duplicables : le contrôle de la généralité des résultats d'une part, leur diffusion d'autre part, s'en verront facilités d'autant.

Ce caractère de duplicabilité peut s'entendre de deux manières :

- Il peut renvoyer à une grande précision sur la description de la situation expérimentale mise en jeu, qui vise à ce que l'on puisse autant que possible reconstituer celle-ci. On voit bien à la fois l'intérêt et les limites d'une telle opération : pas question, évidemment, d'interroger 
strictement les mêmes élèves dans les mêmes conditions météorologiques (mais en général les précisions données ne pèchent pas par excès)...

- Il peut également être lié à des résultats dont on a montré qu'ils sont probablement relativement insensibles à certaines variables de la situation expérimentale, qu'il est intéressant de préciser.

20 Là encore, donc, on n'échappe pas à la nécessité d'un dosage argumenté dans les précisions que l'on donne. Les enseignants de didactique seront par ailleurs reconnaissants aux thésards qui accepteront de mettre en annexe le transcript complet des entretiens: il s'agit là non pas d'une nécessité mais d'un service rendu pour la formation des futurs didacticiens.

21 En matière de conclusions, la difficulté est, bien sûr, de ne pas faire dire plus aux résultats qu'ils ne peuvent le faire, et de se souvenir, en particulier, que corrélation n'est pas cause. Il doit y avoir un rapport étroit entre cette composante et celle qualifiée de "problématique ». Cela semble une trivialité, mais dans la pratique cela se révèle moins évident qu'il n'y parait. Les conclusions négatives ( «telle séquence d'enseignement n'a pas produit les effets attendus ») ou les impossibilités de conclure sont des résultats non seulement avouables mais souvent très utiles. La nécessité, ou l'opportunité, de changer de problématique le sont également. Une thèse qui ne déboucherait "que » sur des questions mieux cernées et des hypothèses affinées peut être tout à fait valable.

\section{Il nous semble souhaitable que la forme prenne en compte :}

La lisibilité et la maniabilité. Une thèse s'adresse d'abord à la communauté des chercheurs et au jury. On utilise un langage spécifique chaque fois que cela s'avère utile, mais on évite l'hermétisme. Sans être forcément complètement et facilement accessible à un professeur non spécialiste, notamment à cause de la problématique quelquefois nouvelle pour lui, la thèse devrait être lisible par tout professeur ou enseignantchercheur motivé, et par un chercheur didacticien étranger parlant français. Enfin, une thèse devrait pouvoir se lire en un temps raisonnable.

\section{On pourrait recommander :}

- que la partie introductive de la thèse (introduction, problématique, état de la question, méthodologie) soit de l'ordre de cinquante pages, plus ou moins une ou deux dizaines de pages : pas davantage.

- que l'ensemble ne dépasse pas (trop) deux cent vingt pages, tout ce qui dépasse devant être mis en annexe.

- qu'un résumé de dix à vingt pages soit rédigé avant soutenance : cela représente certes un surcroît de travail, mais qui faciliterait à la fois la préparation de cette soutenance et la parution rapide d'un article. Pour autant, un tel résumé devrait avant tout donner une idée de l'ensemble de la thèse, ce qui ne coïncide pas nécessairement avec la rédaction prévisionnelle d'un article de revue, lequel peut devoir être plus ciblé.

Dans le même esprit, la présentation de thèses sous forme de collections d'articles assorties d'une synthèse semble hautement souhaitable, mais difficile. Gréa, S. Johsua, R. Journeaux, C. Larcher, R. Lefèvre, J.L. Martinand, G. Rebmann, E. Saltiel, M.G. Séré, J. Thibault, A. Tiberghien, L. Viel, L. Viennot docteurs non encore habilités à diriger des thèses : 
27 M. Goffard, W. Kaminski, L. Maurines, M. Méheut 